\title{
Research on Extraction and Purification of Purple Sweet Potato Anthocyanins
}

\author{
Kaifang Wei ${ }^{1}$, Yichao Duan, Wanwan Zhu, Yunlong Wang, Qiang Ren* \\ ${ }^{1}$ Jining Medical University, Rizhao, Shandong Province, 276826
}

Keywords: extraction; purification; Purple Sweet Potato Anthocyanins

\begin{abstract}
Purple sweet potato is rich in anthocyanins and has a very high use value. The method of extracting anthocyanin from purple sweet potato is analyzed, various extraction methods are compared in detail, and the existing problems in the purification method of anthocyanin are proposed, and the future development direction of purification technology is pointed out.
\end{abstract}

\section{Introduction}

The purple sweet potato is also called black potato, and the potato meat is purple to dark purple. Purple sweet potato is rich in protein and 18 kinds of amino acids that are easily digested and absorbed by the human body. It is also rich in 8 vitamins such as vitamins $\mathrm{C}, \mathrm{B}$ and $\mathrm{A}$, and more than 10 natural mineral elements such as phosphorus and iron. Among them, iron and selenium are abundant, and selenium and iron are essential elements in the body's anti-fatigue, anti-aging and blood-supplementation. In particular, selenium is known as the "anti-cancer king" and is easily absorbed by the body and can be left in the serum to repair the myocardium. Enhance the body immunity, remove free radicals in the body, inhibit the synthesis of DNA in cancer cells and the division and growth of cancer cells, which can prevent the occurrence of cancerous diseases such as gastric cancer and liver cancer. Purple sweet potato is rich in cellulose, which can increase the volume of feces, promote gastrointestinal motility, clean up mucus, gas accumulation, and putrefaction in the intestinal cavity, excrete toxic substances and carcinogens in the stool, keep the stool open, improve the digestive tract environment, and prevent Occurrence of gastrointestinal diseases [1].

\section{Purple Sweet Potato Is Rich in Anthocyanins}

The color of purple sweet potato can be judged to be rich in anthocyanins. The French scientist Dr. Masquarius discovered that anthocyanins are naturally powerful free radical scavengers that can eliminate free radicals in the body. These free radicals are the culprits of aging and cancer. Anthocyanins have preventive and therapeutic effects on over 100 diseases and are known as the 7th essential nutrient following water, protein, fat, carbohydrates, vitamins, and minerals. Anthocyanins are the most direct, effective, and safest free radical scavengers currently found in disease prevention and maintenance of human health. Their ability to scavenge free radicals is 20 times that of vitamin $C$ and 50 times that of vitamin E. Therefore, the nutritional value of purple sweet potato is much higher than other potato.

Purple sweet potato is a special type of sweet potato. Due to its rich red pigment, the sweet potato has attracted the attention of many scholars as an important natural pigment source. Purple Sweet Potato Red Pigment (PSPC) is a natural red pigment leached from the roots, stems and leaves of purple sweet potatoes. It is bright and natural in color, non-toxic, and has no special odor. It has a variety of nutrients, pharmacology and health care functions.

At present, the biggest problem with natural colorants is that they are mostly unstable in the product, and thus affect their application in industry to some extent. It has been reported that acylated pigment molecules can improve the stability of pigments. The purple sweet potato pigment molecule is an acylated pigment molecule, so its stability is strong and its application prospects are extensive [2]. 


\section{Physicochemical Properties of Anthocyanins}

Procyanidins, also known as condensed tannins, are polyphenolic compounds widely found in nature. They have a variety of biological activities. They are highly efficient, low-toxic, and highly bioavailable. They are an extremely strong in vivo activity that has been continuously researched and developed in recent years. Functional factors have been widely used in the fields of food, medicine, cosmetics, etc. The research, utilization, and improvement of botany and agronomic traits related to procyanidins are increasingly receiving attention.

The anthocyanin has a molecular weight of 449.2. Anthocyanins are easily soluble in solvents such as water, methanol and ethanol. Purple sweet potato anthocyanins have good thermal stability, and have good stability below $60{ }^{\circ} \mathrm{C}$, and the preservation rate can reach $100 \%$. Purple sweet potato anthocyanin has strong pressure resistance characteristics, and the preservation rate of anthocyanin remains unchanged after being treated for 15 min under a pressure of $500 \mathrm{MPa}$. The anthocyanin stability of purple sweet potato under different $\mathrm{pH}$ values was different. Under strong acidic conditions $(\mathrm{pH} \leq 3)$, the absorption spectrum of color and characteristics did not change significantly. However, under alkaline conditions, anthocyanins are less stable. Anthocyanins are water-soluble pigments that are red in acid, purple in neutral, and blue or green in alkaline. Purple sweet potato red pigment shows different spectral characteristics in different media. Only in acidic medium does the characteristic absorption peak appear at $530 \mathrm{~nm}$ [3].

Purple sweet potato pigment is sensitive to temperature and light, and is easily decomposed above $60^{\circ} \mathrm{C}$. Daylight and ultraviolet light can also promote its decomposition. Therefore, it should be kept away from light and cool. The $\mathrm{Fe} 3+, \mathrm{Cu} 2+$ and $\mathrm{Al} 3+$ ions had good color-enhancing and color-preserving effects on purple sweet potato pigments. Pb2+ ions had no effect on the stability of purple sweet potato pigments. Zn2+ ions had a great influence on the stability of purple sweet potato pigments. In the study of food additives, oxidants, and reducing agents, it was found that sucrose and sodium benzoate have no effect on the stability of purple sweet potato pigments; NaNO2 has great damage on purple sweet potato pigments; D-sodium erythorbate has good effects on purple sweet potato pigments. Purple sweet potato pigment has good stability to hydrogen peroxide at low concentration and poor stability to high concentration of hydrogen peroxide. Na2SO3 has a great influence on the stability of purple sweet potato pigment. The addition of $\mathrm{Na} 2 \mathrm{SO} 3$ causes a sharp decrease in the absorbance of purple sweet potato pigment.

\section{Use of Anthocyanins}

Anthocyanins usually have the following health functions: Diabetic retinopathy is a sign of diabetes, which is caused by microbleeds in the capillaries of the eye and is a common cause of adult blindness. Anthocyanins can be used to prevent diabetic patients from complications caused by cataract surgery. Edema is caused by the infiltration of water, electrolytes, etc. into the tissues of the human body. It usually manifests as swelling in the injured area. Those who sit for too long have edema, women have edema before menstruation, sports injuries often cause edema, some surgery may have edema, and some diseases can also cause edema. Studies have shown that taking anthocyanins once a day can significantly reduce edema.

Europeans say that anthocyanins are youthful nutrition, skin vitamins, and oral cosmetics. Because it restores collagen vitality, it makes the skin smooth and elastic. Collagen is an essential component of the skin and is a gelatinous substance that makes the body a whole. Vitamin $\mathrm{C}$ is an essential nutrient for biochemical collagen synthesis. Anthocyanin can make more vitamin C effective, which means that vitamin $\mathrm{C}$ can more easily perform all its functions (including the production of collagen). Anthocyanins are linked to collagen and can prevent the damage of collagen-damaging enzymes. Anthocyanins not only help collagen fibers form cross-linked structures, but they can also help restore the damage of excessive cross-links caused by injuries and free radicals. Excessive cross-linking will suffocate and harden connective tissue, causing skin wrinkles and premature aging. Anthocyanins also protect the body from sun damage, prevent radiation damage, and promote healing of psoriasis and skin spots. Anthocyanins are also excellent 
additives for topical skin creams [4].

The purple red sweet potato has good heat resistance and is mainly added to foods as a red to purple coloring agent for foods. Because it is a natural non-toxic product, the amount is not limited to the amount to meet the required degree of coloring. Natural plant extracts have become fashionable as a supplement to regulate body health. Purple sweet potato red pigment has anti-oxidation, anti-tumor and other physiological effects, so it can be used as a functional ingredient of health food.

As purple sweet potato red pigment has anti-oxidation, scavenging free radicals and other effects. Therefore, a skin care product containing a purple sweet potato red pigment can suppress peroxide generation caused by oxygen radicals generated by ultraviolet irradiation, and has a certain effect on improving skin inflammation and anti-oxidation. In addition, it can replace synthetic pigments currently used in the industry and is widely used in cosmetics such as lipsticks, blushers, and shampoos.

\section{Extraction of Anthocyanins}

The extraction of anthocyanins is currently a hot issue in the research and development of anthocyanins, and it is also a key link in the production and use of anthocyanins. In recent years, based on traditional extraction methods, some new technologies or improved extraction methods have also begun to emerge.

\subsection{Organic Solvent Extraction Method}

This is the most widely used extraction method at home and abroad. Most of the mixed solvents such as methanol, ethyl ketone and acetone are used to dissolve and filter the materials, and the anthocyanin in the filtrate is extracted by adjusting the $\mathrm{pH}$ of the solution. Domestic Wu Xinzi extracted with hydrochloric acid - methanol solution, and then with paper chromatography (medium) and column chromatography (polyacetamide) for the separation of anthocyanins. At present, the organic solvent extraction method has been successfully applied to the extraction and separation of most anthocyanin-containing substances such as grape seeds, pomegranate peel and blueberries. The key to organic solvent extraction is the selection of an effective solvent, which requires both a greater solubility of the extracted active ingredient and avoidance of the dissolution of large amounts of impurities. The method is simple in principle and requires less equipment. The disadvantage is that most organic solvents have large toxic and side effects, and the product extraction rate is low [5].

\subsection{Aqueous Solution Extraction Method}

The anthocyanins extracted by organic solvents have many toxic residues and the environmental pollution in the production process is large. In view of this, the aqueous solution extraction method came into being. In this method, plant materials are generally soaked in hot water under normal pressure or high pressure, and then adsorbed by a non-polar macroporous resin, or directly extracted with deoxygenated hot water, then ultrafiltration or reverse osmosis, and concentrated to obtain a crude extract. This method was developed by Duncan and Gilmour in 1998 to extract anthocyanins. The equipment requirements are simple, but the product purity is low. The extraction of natural pigments is usually carried out by soaking, leaching, and percolation of the solvent in the extraction tank under certain temperature conditions. These conventional extraction methods have the advantages of simple process and extraction operation, low equipment investment, and easy production. However, they also have long extraction time, high labor intensity, high energy consumption in raw material pretreatment, and unsatisfactory product quality (poor pigment solubility). Defects such as large changes in color, etc., and large amounts of solvent recovery can easily lead to increased product production costs.

\subsection{Supercritical Fluid Extraction}

Supercritical fluid extraction is the use of pressure and temperature for the extraction of 
supercritical fluids. This method has a high product extraction rate but the equipment cost is too high. Sun Chuanjing studied the extraction of anthocyanins from Ginkgo biloba leaves, blackcurrant seeds and grape seeds using supercritical CO extraction. In this process, $\mathrm{CO}$ and modifiers can be recycled and have no pollution to the environment.

\subsection{Microwave Extraction Method}

This method was first used by Ganzlert E et al. in the separation of various types of compounds in 1986. Li Fengying in China discussed the influence of microwave technology on the extraction amount and molecular structure of proanthocyanidins in grape seeds, which laid the foundation for the research on microwave extraction of active ingredients in grape seeds. The microwave extraction method utilizes the difference in the ability to absorb microwaves in the microwave field to allow certain regions of the matrix material or certain components of the extraction system to be selectively heated, so that the extracted material is separated from the matrix or the system into the in extractive solvents with relatively low dielectric constant and relatively poor microwave absorption. The technology has good selectivity, high extraction rate, high speed, simple operation, and low waste liquid discharge.

\subsection{Ultrasonic Extraction Method}

The ultrasonic extraction method was gradually applied in the chemical and chemical production process after the 1950s, and was mainly concentrated in the research fields of the composition of plant medicines, polysaccharides, and extraction of other functional components. Ultrasonic extraction method has a good prospect, simple operation, rapid and high efficiency, and clean and pollution-free production process. In 2008, comparative experiments conducted by Corrales et al. on the effect of different extraction methods on the extraction rate of anthocyanins in grapes showed that the ultrasonic-assisted extraction of phenols such as anthocyanins was more efficient than extraction with hot dip $70^{\circ} \mathrm{C}$ under the same conditions.

\section{Anthocyanin Purification Method}

\subsection{Macroporous Resin Adsorption Chromatography}

Macroporous adsorption resin is a separation material combining the adsorption and screening principles. It is a type of organic polymer adsorbent developed in the past 10 years. Its adsorption is essentially the surface of the surface molecule due to uneven force. Adsorption phenomenon, this adsorption is due to van der Waals attraction or the result of hydrogen bonding, and because of the porous structure of macroporous adsorption resin, it has a screening effect on substances with different molecular sizes. Through this adsorption and screening, organic compounds can be separated, purified, purified, concentrated, and other different purposes by eluting a certain solvent according to the difference in the adsorptive power and the size of the molecular weight. In general, for non-polar macroporous resins, the smaller the eluent polarity, the stronger the elution ability; for polar macroporous resins and more polar compounds, the use of polar solvents can be better.

\subsection{Ion Exchange Resin Chromatography}

The ion exchange column chromatography is a chromatographic separation method in which an ion exchange resin is used as the stationary phase. This method allows the exchangeable ions carried by the functional groups immobilized on the polymer skeleton to dissociate in solution and can be in a larger range, it is free to move and diffuse into the solution, and the same type of ions in the solution can also diffuse from the solution into the network and pores of the polymer. When the concentration difference between these two ions is large, it will produce A driving force of exchange makes exchanges occur between them. The greater the difference in concentration, the faster the exchange rate, and the use of the driving force of this concentration difference to cause a reversible exchange reaction of the exchangeable ions on the resin. 


\subsection{Gel Chromatography}

Gel chromatography, also known as exclusion chromatography, works on the principle of using the molecular sieves of a gel with a network structure to separate the molecules according to their molecular size. Chromatographically separated stationary phases are generally cross-linked polysaccharides (eg, dextran or agarose), small molecules can enter the interior, elute slowly, and macromolecules are excluded from the outside. The speed of the down is fast. When the mixed solution passes through the gel filtration column, the substances in the solution are separated by different molecular weight sieves. The outstanding advantages of gel column chromatography are that the gels used for chromatography are inert, non-charged, weak in adsorption, and relatively mild in operating conditions. They can be performed over a wide range of temperatures, do not require organic solvents, and require separation. The physicochemical properties of the ingredients can be better maintained.

\section{Conclusion}

Therefore, under the premise of ensuring food safety, how to develop a highly efficient and green method for extracting and refining purple anthocyanins, as well as trials and the use of these methods to extract purple anthocyanins, and further study and evaluate the anthocyanin of purple sweet potato The effects of human body and health care will play a positive role in promoting people's understanding of the efficacy of purple anthocyanin and driving the development of purple potato industry.

\section{References}

[1] Xu Gang. Extraction and Antioxidation of Flavonoids from Sweet Potatoes [J]. Journal of Food and Biotechnology. 2007 (04).12

[2] LUO Jun, ZHANG Xueying, LI Guangping. Determination of anthocyanin in fruits by HPLC [J]. Shanghai Journal of Agricultural Sciences. 2006 (03).87

[3] Qin Huimin, Zhu Siming, Yu Shujuan. Antibacterial and Antioxidant Activity of Hesperidin and Copper Complexes [J]. Food Science and Technology. 2006 (06).69

[4] Zhu Hongmei, Han Yongbin, Gu Zhenxin, Fan Gongjian. Study on Adsorption and Desorption of Purple Sweet Potato Pigment with Macroporous Resin [J]. Chinese Journal of Agricultural Engineering. 2006 (05).22

[5] Sun Shiping, Dai Bin, Hong Chenglin, Tian Liping. Research progress of chromatographic determination of beet red pigment [J]. China Food Additives. 2006 (03).159 